\title{
Cross-culture Business Communication by Emoji in GMS
}

\author{
Feng Yuhui* \\ International Business School \\ Yunnan University of Finance and Economic \\ Kunming, China \\ feng@ynufe.edu.cn \\ Qiu Menlan \\ International Business School \\ Yunnan University of Finance and Economic \\ Kunming, China \\ 879579662@qq.com
}

\author{
Li Yan \\ International Business School \\ Yunnan University of Finance and Economic \\ Kunming, China \\ cyliyan2002@163.com \\ Yang Haiyang \\ International Business School \\ Yunnan University of Finance and Economic \\ Kunming, China \\ frank2h@foxmail.com
}

\begin{abstract}
Emoji has developed rapidly on Internet in recent years and plays an important role in cross-cultural communication. But whether it can be adopted in the communication by people in Southeast Asia remains a problem. Furthermore, whether graphical symbols like emoji are capable of working as a pre-trade supplementary approach of information exchange in GMS area, considering limitations like agriculture-led population and diverse minority languages. Besides, English is not universally adopted in this area, so it's hard for people there to use English as an intermediate language. To respond to this question, we conducted two Web-based experiments through six groups of participants, in some Southeast Asia countries. The results showed that the people in South \& Southeast Asia are able to share their feelings through common emoji on Internet. Beyond that, some statements consist of extended and intelligible graphical symbols with a specific rule can also convey business information.
\end{abstract}

Keywords-emoticons; graphic symbols; cross-cultural communication; business communication

\section{INTRODUCTION}

In recent years, the rapid development of emoji became more and more popular on Internet. The Emoji ideograph for Heart (and Love) is the Top Word for 2014 according to the 15 th annual survey of the English language by the Global Language Monitor. The Heart and Love emoji, emoticon, and its variations thereof appear billions of times a day around the world - across languages and cultures. This is the first time an ideograph has captured Word of the Year honors (Global Language Monitor, 2014) [1]. Xu Bing (2012) wrote a novel to the book: from point to point, described one day in the life of white-collar workers with pure graphical symbols [2]. Apple's character set of mobile operating system from iOS8.3 until to 9.1 contains approximately 184 or more graphic symbols. Also in Google's Android 6.0.1, Microsoft Windows10 added a lot of graphic symbols. The popularity of emoticons in most countries has undoubtedly facilitated cross-cultural communication. Most people in the world can share their feelings with a universal set of emoji. However, in this research, we focus on examining whether it is possible to use emoji for business communication in Southeast Asia where individuals share different cultural backgrounds.

As a newly approach of trading, cross-border E-commerce became more and more popular and has infiltrate into all areas of international business activities. It has a profound impact on changing the international economic landscape. Cross-cultural instant communication was one kind of conspicuous obstacle in the development of cross-border E-commerce, particularly in multi-ethnic and multi-lingual areas. For example, GMS(Great Mekong Subregion Cooperation) is consist of nations and regions include Cambodia, Vietnam, Laos, Myanmar, Thailand, Yunnan province of China also included in this area, and there are 7 different official languages in this region (Table1). Yunnan especially, 27 different languages are used by 25 ethnic group, and Guanxi province, 15 different languages are used by 12 ethnic group (Table 2), most of them are minority languages (Yuhui Feng, 2014) [3].

\section{TABLE I. LANGUAges USED IN SOUTHEAST AsIA}

\begin{tabular}{ll}
\hline Countries & Languages \\
\hline Vietnam & Vietnamese, French, Khmer \\
Laos & Lao, French \\
Cambodia & Khmer, French \\
Myanmar & Burmese, Other minority languages \\
Thailand & Thai \\
China & Chinese \\
\hline
\end{tabular}

\footnotetext{
* Corresponding author
} 
TABLE II. MinORity LANGUAgEs Used In YUnNAN AND GUANGXI

\begin{tabular}{|c|c|c|c|}
\hline Nationality & Languages & Nationality & Languages \\
\hline Yi Nationality & Sani Language & Wa Nationality & Wa Language \\
\hline Bai Nationality & Bai Language & Naxi Nationality & Naxi Language \\
\hline Jingpo & Jingpo:Jingpo & Yao Nationality & Mian:Mian \\
\hline Nationality & $\begin{array}{l}\text { Language; } \\
\text { Zaiwa: } \quad \text { Zaiwa } \\
\text { Language }\end{array}$ & & $\begin{array}{l}\text { Language; } \\
\text { Bunu:Bunu } \\
\text { Language }\end{array}$ \\
\hline Dai Nationality & Dai Language & Hani Nationality & Hani Language \\
\hline $\begin{array}{l}\text { Zhuang } \\
\text { Nationality }\end{array}$ & Zhuang Language & Zang Nationality & Zang Language \\
\hline Miao Nationality & Miao Language & $\begin{array}{l}\text { Bulang } \\
\text { Nationality }\end{array}$ & Bulang Language \\
\hline Lisu Nationality & Lisu Language & Buyi Nationality & Buyi Language \\
\hline $\begin{array}{l}\text { Deang } \\
\text { Nationality }\end{array}$ & Deang Language & $\begin{array}{l}\text { Jinuo } \\
\text { Nationality }\end{array}$ & Jinuo Language \\
\hline Nu Nationality & $\begin{array}{l}\text { Bijiangnusu:Nusu } \\
\text { Language; } \\
\text { Lanping,Lushui,Rour } \\
\text { uo;Rouruo Language } \\
\text { Fugong,Anong:Anon } \\
\text { g Language }\end{array}$ & $\begin{array}{l}\text { Menggu } \\
\text { Nationality }\end{array}$ & Menggu Language \\
\hline Shui Nationality & Chinese & Man Nationality & Chinese \\
\hline Hui Nationality & $\begin{array}{l}\text { Chinese,Arabic(Chil } \\
\text { dren scriptures) }\end{array}$ & Pumi Nationality & Pumi Language \\
\hline Lahu Nationality & Lahu Language & $\begin{array}{l}\text { Achang } \\
\text { Nationality }\end{array}$ & Achang Language \\
\hline
\end{tabular}

Regarding the language barrier on cross-border Ecommerce websites, 3 different solutions are generally adopted for now, they are: (1) Multi-language pages, (2) Web translation through software, (3) Artificial asynchronous \& synchronous translation. None of these solutions are perfect. Solution (1) is known as static pages which mainly for press releases and difficult to conduct interactive E-commerce businesses. Solution (2) has two obvious flaws while one is the accuracy limitation using translation software (roughly an accuracy of $70 \%$ ), the other is the lack of translation support for minor languages such as Khmer, Lao, Burmese, etc. Solution (3) has problems like cost effectiveness, lack of immediacy and no guarantee of long-term quality services. In addition, due to the low rate of higher education, there are not much applicability for English in this area.

Therefore, in a web-based context, it is hard for the people in GMS areas to communicate in English as an intermediary language. Moreover, translation software cannot cover all the minority languages. So we attempted to shed light on the question whether graphical symbols like emoji are capable of working as a pre-trade supplementary approach of information exchange.

\section{LITERATURE REVIEW}

Walther (1992) conducted a research using SIP (social information processing) and found the replicability of building human relations in network environment [4]. With the increase of technology development and network usage, people have changed their tactics to adapt to this communication environment. Walther \& Tidwell (1995) argued that given the non-verbal expression capabilities we have, people gradually learned good communication skills [5]. Tidwell \& Walther (2002) considered that by changing the social strategy, it is possible to manage interpersonal relationships [6]. Theory suggests that the text-based traditional computer-mediated communication (CMC) unlike in face to face (F2F) communication.F2F can obtain more important information from human or non-verbal language, like expression, attitude personality companions. But CMC can get information from feelings symbols by non-verbal cues now (Dresner,2010; Rice,1987; Crystal,2001,Derks,2007; Walthe,2001; Kiesler,1984; Liang Xiaoyan,2014; Ye Yun,2013) [7-13]. Emoji have been widely disseminated across the globe, and to a certain standard written in Unicode, an international generalpurpose computing character encoding, in the mail, Web, mobile phones and computer operating systems.

Studies have shown that graphic symbols play a role in the cross-cultural communication under different cultural backgrounds. Ekman (1971) demonstrated by experiments that people from different culture have a similar recognition of emotional facial expressions [14]. They choose 319 adults and children who have had minimal visual contact with literate cultures in New Guinea and showed the subjects a group of three pictures simultaneously. Then they read a story and told the subjects to point to the picture in which the person's face showed the emotion described in the story. The results provide evidence in support of that the association between particular facial muscular patterns and discrete emotions is universal. Piamonte (2001) studied the identification bias of graphic symbols in different cultural backgrounds [15]. The experiments instead showed a high level of correct symbol perception. Tomkins (1962) assumed the existence of 8 primitive emotions which are interest, joy, surprise, pain, fear, shame, contempt and anger [16]. Izard (1968) set a criteria including 9 basic expressive emotions on the basis of earlier work and built a set of emotional test [17]. He then presented it to subjects from the United States, UK, France and Greece, and resulted in an accuracy rate of 60\%. Psychologist Paul Ekman did an experiment by showing some photographs of facial expressions to isolated tribal people. These people can accurately understand the meaning of these expressions. On the contrary, their facial expressions can also be correctly read by people from civilized world. Despite a small difference, this means the use and understanding of human expressions are identical.

There are not much awareness of whether the application of Emoji has come to an end. Dresner (2010) discussed the complementary function of emoticons in $\mathrm{CMC}$ and he considered that emoticons can not only express feelings but also have a pragmatic meaning of extralinguistic [7]. Mingxia He believes emoticons and instant messaging (IM) have a mutual influence and emoticons would bring great commercial value, due to the IM platform enhancement and also the selfevolution of the emoticons [18].

In summary, emoji are widely used to express emotions through Internet and make up for the lack of nonverbal cues. Previous studies have made it clear that emoji plays an important role in cross-cultural communication, but similar application has not been verified in Southeast Asia. Not to mention the business application of emoji, hardly anyone has filled the void before. 


\section{HYPOTHESES}

From the studies and needs mentioned above, we aim to do further research on cross-cultural communication and business application in Southeast Asia. Thus we propose the following hypotheses:

H1: People from South \& Southeast Asia can exchange feelings cross-culture with common emoji in Internet.

H2: The business information can be convey by graphical symbols plus some rule group using extended and easy to understand.

\section{EXPERIMENTS}

Two experiments have been designed to verify two hypotheses.

We choose the method of online experiments due to the involvement of several Southeast Asian countries. According to the requirements from the designed experiments, we use http protocol, html, JavaScript and other Web languages techniques. Then design corresponding Web pages and connect them with a server. Subjects participate in the experiment using their native languages with PCs (or smartphones, tablets). They are asked to choose several answers to the questions showing on the page and the system records their operations and transmits the collected data to the database server, and then comes the statistical analysis. We choose to use online experiment because it agrees with actual situation, has larger sampling space, low cost and high efficiency.

Experimental emotional symbols choice from seven kinds of typical emotional, i.e. happiness, anger, worry, anxiety, sadness, fear, surprise.

\section{A. Experiment 1 (verification hypotheses H1)}

\section{1) Experimental Design}

In order to provide evidence for $\mathrm{H} 1$, we try to understand whether there is consistent understanding of a set of emotional emoji from people in China and Southeast Asia.

We hope subjects are capable of identifying these emoji without processing. According to Simon (1974, pp 183) of the null hypothesis, that the mean values of the dependent variables were not significantly different for sets of subjects run under different experimental conditions [19]. If the data led to rejection of the null hypothesis, one bit of information had been obtained-that the dependent variable was, apparently, affected by the independent variable.

So we use spontaneous identification in experimental group and cued-response in control group. If there is no significant difference between the two groups, then Hypothesis 1 is valid. The control group was divided into three groups. The first group is given one correct emoji and several interference emoji, subjects shall select an emoji based on the text tips given. The second group choose an emoji and make a single selection from multiple text answers. The third group is given a tip word and select one emoji that best match the tip word.

\section{2) Participants}

Subjects come from GMS countries including Laos, Myanmar, Thailand and Vietnam and someone who were able to mobilize in the host country. They were divided into four groups: exp1a as the experimental group, exp1b, exp1c and expld as control groups. When subjects login the website, they are randomly allocate to a group by the computer according to each group number in the database.

\section{3)Procedures}

Experimental group expla are given emoji one by one to identify shown in Table 3 and fill in the answers with characters until they finish identifying all seven emoji.

TABLE III. EXPERIMENTAL MATERIALS OF GROUP EXP1A

\begin{tabular}{|c|c|c|c|c|c|c|}
\hline $\begin{array}{l}\text { hap- } \\
\text { pyness }\end{array}$ & $\begin{array}{l}\text { an- } \\
\text { ger }\end{array}$ & worry & $\begin{array}{l}\text { anxi- } \\
\text { ety }\end{array}$ & $\begin{array}{l}\text { sad- } \\
\text { ness }\end{array}$ & fear & $\begin{array}{l}\text { sur- } \\
\text { prise }\end{array}$ \\
\hline & & & & & .. & $(\%)$ \\
\hline
\end{tabular}

The control group was divided into 3 groups:

a) Control group explb was given one specific emoji shown in and several interference emoji shown in Table 4. One tip word was given as well. They were required to choose the most suitable emoji and continue to do so until they finish all 7 emoji.

TABLE IV. EXPERIMENTAL MATERIALS OF GROUP EXP1B

\begin{tabular}{|c|c|c|c|c|c|c|}
\hline $\begin{array}{l}\text { hap- } \\
\text { pyness }\end{array}$ & anger & worry & anxiety & sadness & fear & $\begin{array}{l}\text { sur- } \\
\text { prise }\end{array}$ \\
\hline & 68 & & 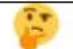 & $\because$ & 67 & \\
\hline (90) & (20) & 8 & 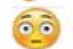 & (20) & 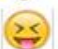 & \\
\hline 8 & 60 & & i. & 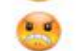 & $\underline{-}$ & \\
\hline$=$ & 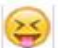 & ㅇ. & 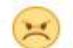 &  & $\because$ & \\
\hline
\end{tabular}

b) Control group exp1c was given one specific emoji shown in Table5 and multiple text selections. They were required to make a single selection one by one until they finish all 7 emoji.

TABLE V. EXPERIMENTAL MATERIALS OF GROUP EXP1C

\begin{tabular}{|c|c|c|c|c|c|c|}
\hline $\begin{array}{l}\text { hap- } \\
\text { pyness }\end{array}$ & anger & worry & $\begin{array}{l}\text { anxi- } \\
\text { ety }\end{array}$ & $\begin{array}{l}\text { sad- } \\
\text { ness }\end{array}$ & fear & $\begin{array}{l}\text { sur- } \\
\text { prise }\end{array}$ \\
\hline & 76 & 6 & $\tau$ & & & \\
\hline
\end{tabular}

c) Control group exp1d was given one tip word and several emoji shown in Table 6. They were required to choose one emoji that best match the tip word and continue to do so until all 7 emoji are finished.

TABLE VI. EXPERIMENTAL MATERIALS OF GROUP EXP1D

\begin{tabular}{|c|c|c|c|c|c|c|}
\hline $\begin{array}{l}\text { hap- } \\
\text { pyness }\end{array}$ & anger & worry & $\begin{array}{c}\text { anxi- } \\
\text { ety }\end{array}$ & $\begin{array}{l}\text { sad- } \\
\text { ness }\end{array}$ & fear & $\begin{array}{l}\text { sur- } \\
\text { prise }\end{array}$ \\
\hline (1) & 08 & 10 & 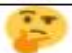 & & & \\
\hline$\theta$ & $\cdots$ & $\Leftrightarrow \infty$ & 93 & 25 & & (a) \\
\hline 5 & -5 & (53) & उहु) & 0 & 0 & 60 \\
\hline
\end{tabular}




\section{B. Experiment 2 (verification Hypothesis 2)}

\section{1) Experimental Design}

In order to verify Hypothesis 2, we create statements consisting of multiple graphical symbols to see if it is capable of conveying business signals.

When subjects login the website, they are randomly allocate to a group by the computer.

\section{2) Participants}

Subjects were divided into two groups which are exp2a (experimental group) and exp2b (control group).

\section{3) Procedures}

a) Experimental group exp $2 a$

First is a learning progress.

(1) Learning of the narrative materials consist of graphical symbols. Subjects are asked to learn the dialogues written in graphical symbols by Xu Bing (as shown in Fig 1). This text has a hidden translation. If the subjects cannot get the meaning of Fig 1, a [Translation] button below can be click to show the hidden translation (as shown in Fig 2).

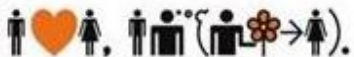

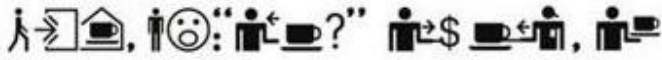 $\hat{k \rightarrow \rightarrow} \rightarrow$ nin.}

\section{tiñ}

Fig. 1. Learn to communication with graphical symbols

1, he loved her, he wanted to give her a flower.
2, he went to the coffee shop called the cup of coffee.
After pay, with the coffee and sat down.
3, he sent her a letter.

Fig. 2. Learn the translation of the materials

Tip: Graphical symbols have the following rules:

\begin{tabular}{|c|}
\hline 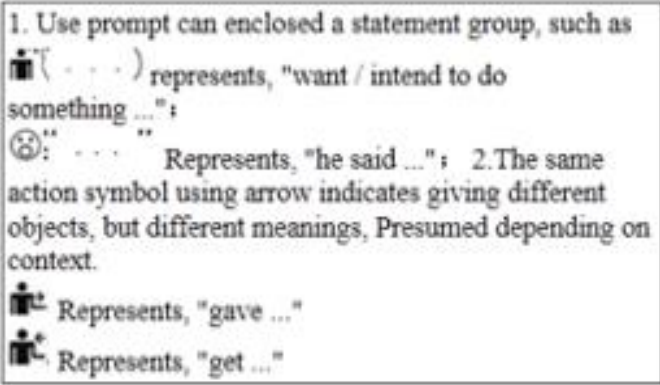 \\
\hline
\end{tabular}

Fig. 3. Learning some rules

Finally, start the experiment. A dialogue consists of graphical symbols is given below and subjects were asked to answer the overall meaning and the meaning of each sentence shown in Fig 4.

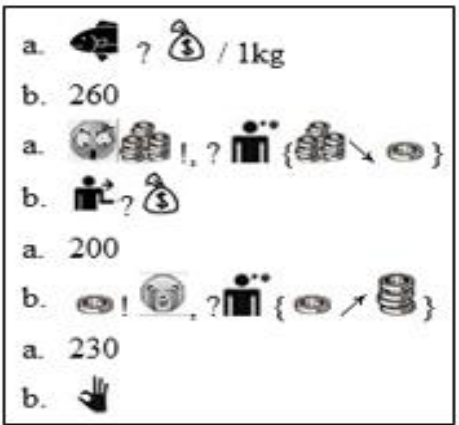

Fig. 4. Dialogue

Note: The meaning of the graphical symbols is as follows (invisible to the subjects)
a. How much is $1 \mathrm{~kg}$ of fish?
b. 260
a. (frightened) it is too expensive, can you lower the price?
b. How much would you like?
a. 200
b. (sad) Price is too low, a little more to it.
a. 230

\section{b) Control group exp $2 b$}

This group haven't to learn and tips section. Done directly Fig 4 recognition.

\section{ANALYSES AND RESULTS}

\section{A. Measures}

Correct recognition rate can be considered the percentage of correctly identified individuals accounted for the overall. We try to determine people's level of acceptance toward emoji by calculating correct recognition rate of emoji in each experiment The higher the correct recognition rate is, the more consistent the lever of acceptance is.

After gathering the statistical information from experiment 1, the correct recognition rate of seven emoji is shown in Table 7.After gathering the statistical information from experiment 1 , the correct recognition rate of seven emoji is shown in Table 7.

TABLE VII. CORRECT RECOGNITION RATE OF EXPERIMENT 1

\begin{tabular}{cccccccccc}
\hline \multicolumn{2}{c}{$\begin{array}{c}\text { happine } \\
\text { ss }\end{array}$} & anger & worry & \multicolumn{2}{c}{ anxiety sadness } & fear & \multicolumn{2}{c}{ surprise $\operatorname{arr}(\%)$} \\
\hline exp1a & 90.00 & 100.00 & 90.00 & 60.00 & 100.00 & 70.00 & 90.00 & 85.71 \\
exp1b & 55.56 & 100.00 & 100.00 & 100.00 & 100.00 & 100.00 & 100.00 & 93.65 \\
exp1c & 85.71 & 100.00 & 85.71 & 85.71 & 85.71 & 85.71 & 85.71 & 87.76 \\
exp1d & 83.33 & 83.33 & 83.33 & 83.33 & 83.33 & 100.00 & 83.33 & 85.71 \\
arr(\%) & 78.65 & 95.83 & 89.76 & 82.26 & 92.26 & 88.93 & 89.76 & $\mathbf{8 8 . 2 1}$ \\
\hline
\end{tabular}

Note: average recognition rate $=$ ar

The table shows the average recognition rate which from exp1a, exp1b, exp1c, exp1d in experiment 1 are $85.71 \%$, $93.65 \%, 87.76 \%$ and $85.71 \%$, the average is $88.21 \%$. Exp $1 \mathrm{~b}$ has the highest recognition rate because it is given the most symbols and clues. The difference between other groups was not significant. 
Subjects' average recognition rates in experiment 1 which include the recognition of seven kinds of emoji (happy, anger, worry, anxiety, sad, fear and surprise) are $78.65 \%, 95.83 \%$, $89.76 \%, 82.26 \%, 92.26 \%, 88.93 \%$ and $82.26 \%$, the average recognition rate is $88.21 \%$. Anger and sadness had higher recognition rate while the recognition rate of happy is relatively low, perhaps due to some sort of sadness that Asians are born with.

Two sets of data from experiment 2 were collected and subjects' correct recognition rates are shown in Table 8 .

TABLE VIII. DATA OF EXPERIMENT 2

\begin{tabular}{lcccccccccc}
\hline & Q & Q1 & Q2 & Q3 & Q4 & Q5 & Q6 & Q7 & Q8 & $\begin{array}{c}\operatorname{arr} \\
\text { \%) }\end{array}$ \\
\hline exp2a & 87.67 & 86.67 & 84.67 & 88.54 & 82.62 & 83.46 & 86.67 & 87.67 & 86.67 & 86.07 \\
exp2b & 87.55 & 86.57 & 83.52 & 87.09 & 82.16 & 83.07 & 85.50 & 86.88 & 85.87 & 85.36 \\
Difference & 0.12 & 0.10 & 1.15 & 1.45 & 0.46 & 0.39 & 1.17 & 0.79 & 0.80 & 0.71 \\
\hline
\end{tabular}

Average recognition correct rate of test group exp2a is 86.07, while control group exp2b is 86.07. Small differences are shown between the two groups and this indicate that the case content is in line with people's life experience.

By analyzing the enhance level of the recognition rate between control group and experimental group, we found that learning to improve the correct recognition rate is insignificant. (Results are shown in Table 9).

TABLE IX. ANALYSIS THE INCREASE DEgREE OF RELATIVE RECOGNITION RATE OF CONTROL GROUP TO EXPERIMENTAL GROUP

\begin{tabular}{|c|c|c|c|}
\hline not significant almost not & not sure & little & significantly \\
\hline $\mathrm{Q}$ & $\sqrt{ }$ & & \\
\hline Q1 & $\sqrt{ }$ & & \\
\hline Q2 & & & $\sqrt{ }$ \\
\hline Q3 & & & $\sqrt{ }$ \\
\hline Q4 & & $\sqrt{ }$ & \\
\hline Q5 & & $\sqrt{ }$ & \\
\hline Q6 & & & $\sqrt{ }$ \\
\hline Q7 & & $\sqrt{ }$ & \\
\hline Q8 & & $\sqrt{ }$ & \\
\hline arr & & $\sqrt{ }$ & \\
\hline
\end{tabular}

\section{B. Results}

Expla of Experiment 1 is the test group and reached the correct recognition rate of 85.71 without any learning process or tips. Exp1b reached 93.65 with more symbols clues. Exp1c is an experiment with one symbol and multiple text tips, it reached 87.76. Exp1d only got text tips and reached 85.71. There is no significant difference in four groups. If the average of the dependent variables are not significantly diffident and the data collected reject the null hypothesis, then we can say the experimenter has got a reliable conclusion that the dependent variable is significantly affected by the independent variable (the correct recognition rate) (Simon 1974) [19]. So what the data showed supported H1: People from South \& Southeast Asia can exchange feelings cross-culture with common emoji in Internet.

Experiment 2 is designed to determine whether business information can be conveyed by extended and understandable sentences using graphical symbols. Subjects are going to determine the hidden meaning of the sentences by correctly interpreting the symbols and the context. Final results showed that the subjects from control group reached a recognition rate of 86.01 without any learning process or clues. While after a period of learning, the recognition rate of subjects from text group has slightly improved and reached 86.67. These results supported our hypothesis $\mathrm{H} 2$ that the business information can be convey by graphical symbols group using extended and easy to understand plus some rule.

\section{DISCUSSION AND CONCLUSION}

What we did in our experiments supported two hypothesis. We choose some basic emoji which people are most familiar with and besides, the most common scenes for the commercial application of the extended symbols. The results showed that there is not much barriers of communication in GMS area, even with some complex contextual factors.

We aim to conduct research on more emoji in the future, such as the eight kind of primitive emotions (Tomkins, 1970) [16], and 9 kinds of basic emotions (Izard, 1968) [17].

More communication scenarios can be set and more complex statements can be researched. More effective rules can be absorbed from psychology, graphic design and computer intelligent. We believe that by going through a specific learning process, people can eventually reach a certain level of cross-cultural business communication.

\section{ACKNOWLEDGEMENT}

This research was sponsored by the Humanities and Social Sciences General Project (Research on methods of nonverbal symbol instant communication in GMS, grant 11XJA870001) commissioned by China's Ministry of Education.

\section{REFERENCES}

[1] “Global Language Monitor,” Top words of 2014, December 2014.

[2] B. Xu, "To Book: from point to point," Guilin: Guangxi normal university press, 2012.

[3] Y.H. Feng and M.Y. Hua, "Research on Non-verbal Graphic Symbol Communication of Cross-Border e-Commerce," Digital Services and Information Intelligence. Springer Berlin Heidelberg, 2014, pp. 251-263.

[4] J.B. Walther, "Interpersonal effects in computer-mediated interaction: A relational perspective," Communication Research, 1992, vol. 19, pp. 5290.

[5] J.B. Walther, and L.C. Tidwell, "Nonverbal cues in computer-mediated communication and the effect of chronemics on relational communication," Journal of Organizational Computing and Electronic Commerce, vol. 5, 1995, pp. 355-378.

[6] L.C. Tidwell, and J.B. Walther, "Computer-mediated communication effects on disclosure,impressions and interpersonal evaluations: Getting to know one another a bit at a time," Human Communication Research, vol. 25, 2002, pp. 317-348.

[7] E. Dresner and S.C. Herring, "Functions of the nonverbal in CMC: Emoticons and illocutionary force," Communication theory, vol. 20, 2010, pp. 249-268.

[8] R.E. Rice and G. Love, "Electronic emotion socioemotional content in a computer-mediated communication network," Communication research, vol. 14, 1987, pp. 85-108.

[9] D. Crystal, "Language and the Internet," Cambridge University Press, 2001 . 
[10] J.B. Walther, and K.P. D'Addario, "The impacts of emoticons on message interpretation in computer-mediated communication," Social science computer review, vol. 19, 2001, pp. 324-347.

[11] S. Kiesler, J. Siegel, and T.W. McGuire, "Social psychological aspects of computer-mediated communication," American psychologist, vol. 39 1984, pp. 1123.

[12] X.Y. Liang, S.Q. Wang, A.H. Yao, and D.Q. Liang, "Emoticons: nonverbal line in network space," Science of social psychology, 2014, pp. 29-34+98.

[13] Y. Ye, "Internet emoticons popular, change and space extended," Shanghai normal university, 2013.

[14] P. Ekman, and W.V. Friesen, "Constants across cultures in the face and emotion," Journal of personality and social psychology, 1971, 2, vol. 17, pp. 124.
[15] D.P.T. Piamonte, J.D.A. Abeysekera, and K. Ohlsson, "Understanding small graphical symbols: a cross-cultural study," International Journal of Industrial Ergonomics, vol. 27, 2001, pp. 399-404.

[16] S.S. Tomkins, "Affect, imagery, consciousness: The positive affects," vol. 1, 1962.

[17] C.E. Izard, "The emotions as a culture-common framework of motivational experiences and communicative cues," Technical Report Vander-ilt University, Contract Nonr, 1968, Office of Naval Research.

[18] M.X. He, "Emotional symbol commercial value under the network instant messaging environment promotion," Journal of art and design (theory), 2011, pp. 49-51

[19] H.A. Simon, "How big is a chunk?" Science, vol. 4124, 1974, pp. $482-$ 488 . 